\title{
Von Willebrand factor (vWF): marker of endothelial damage and thrombotic risk in COVID-19?
}

\author{
Authors: Eleni E Ladikou, ${ }^{\mathrm{A}}$ Helena Sivaloganathan, ${ }^{\mathrm{A}}$ Kate M Milne, ${ }^{\mathrm{A}}$ William E Arter, ${ }^{\mathrm{B}}$ Roshan Ramasamy, \\ Ramy Saad, ${ }^{D}$ Simon M Stoneham, ${ }^{A}$ Barbara Philips, ${ }^{\mathrm{E}}$ Alice $\mathrm{C}$ Eziefula ${ }^{\mathrm{F}}$ and Timothy Chevassut $^{\mathrm{G}}$
}

\section{Background}

COVID-19 infection is characterised, among other features, by a prothrombotic state with high rate of venous thromboembolism (VTE), D-dimer, and fibrinogen levels. Clinical observations have also highlighted that these patients have elevated von Willebrand factor (vWF) and factor VIIIc.

\section{Methods}

24 consecutive COVID-19 positive patients were selected from the intensive care unit (ICU) or the high acuity ward of Brighton and Sussex University Hospitals NHS Trust.

Results

The rate of VTE was $25 \%$ and mortality rate was $16.7 \%$.

Fibrinogen and D-Dimers were elevated, 7.9 (1.6) g/L and 2.4 (2.02) $\mathrm{ug} / \mathrm{ml}$ respectively. Factor VIIIc and von vWF antigen levels were both extremely elevated at 279 (148) u/dL and 350 (131) \% respectively, which are comparable to levels seen in ICU patients with severe sepsis. vWF levels were significantly higher in patients that died $(p=0.017)$ and showed a positive correlation with age. There was a statistically significant association between COVID-19 disease and non-O blood group $(p=0.02) ; 80 \%(4 / 5)$ of COVID-19 patients with VTE were blood group $\mathrm{A}$.

\section{Conclusion}

Very high levels of vWF and factor VIIIc are common in COVID-19 patients, comparable to levels in severely septic non-COVID ICU patients. This could contribute to the

Authors: ${ }^{A}$ academic clinical fellow, Brighton and Sussex University Hospitals NHS Trust, Brighton, UK and Brighton and Sussex Medical School, Falmer, UK; B post-doctoral research fellow, Department of Chemistry, University of Cambridge, Cambridge, UK; ' Cacute care common stem (ACCS) trainee, Brighton and Sussex University Hospitals NHS Trust, Brighton, UK; Dinternal medicine trainee (IMT), Brighton and Sussex University Hospitals NHS Trust, Brighton, UK; Ereader in intensive care medicine, Brighton and Sussex Medical School, Falmer, UK and Brighton and Sussex University Hospitals NHS Trust, Brighton, UK; F senior lecturer and consultant in infectious disease, Brighton and Sussex University Hospitals NHS Trust, Brighton, UK and Brighton and Sussex Medical School, Falmer, UK; Greader in haematology and director of academic training, Brighton and Sussex Medical School, Falmer, UK and consultant haematologist, Royal Sussex County Hospital, Brighton, UK hypercoagulable state and increased VTE rate in COVID-19. Further studies are needed to evaluate the use of vWF for stratifying thrombotic risk in COVID-19 and to determine if elevated vWF is contributing to disease pathogenesis.

KEYWORDS: von Willebrand factor, factor VIIIc, COVID-19, venous thromboembolism, blood group

DOI: $10.7861 /$ clinmed.2020-0346

\section{Introduction}

Severe acute respiratory syndrome coronavirus 2 (SARS-CoV-2), causing coronavirus disease 2019 (COVID-19), has infected more than 12 million people and caused more than 500,000 deaths worldwide. 'It affects primarily the respiratory system, but can also involve the cardiovascular, gastrointestinal and haematological systems. Autopsy reports of COVID-19 cases have revealed alveolar exudative inflammation, epithelium proliferation, interstitial inflammation and hyaline membrane formation in the lungs. Pathological lesions were also seen in other organs such as heart, vessels, liver and kidney. ${ }^{2}$ The most characteristic finding in non-survivors with COVID-19 was diffuse alveolar damage, which was accompanied by extensive microvascular thrombosis in lungs and extrapulmonary organs. ${ }^{3}$ Megakaryocytes have been identified within the small vessels and alveolar capillaries identified by CD61 and vWF immunostains. ${ }^{4}$

The rate of venous thromboembolism (VTE) in critically ill hospitalised patients is $10 \% .{ }^{5}$ However, early studies show that the incidence of VTE in patients with severe novel coronavirus pneumonia can be as high as $25 \%{ }^{6}$ Early case reports have described an association between COVID-19 and venous thrombo-embolic events.?

\section{Key points}

> COVID-19 is associated with a hypercoagulable state with increased VTE rate.

> COVID-19 patients frequently have very high von Willebrand factor (vWF) and factor VIIIc levels that are comparable to those seen in ICU patients with severe sepsis.

> Further studies are needed urgently to evaluate the use of VWF as a pragmatic measure to stratify thrombotic risk in COVID-19. 
Tang et al found that treatment with prophylactic heparin (lower molecular weight heparin or unfractionated heparin) was associated with a reduced 28-day mortality in severe COVID-19.8

Coagulopathy is a hallmark of severe COVID-19, with $71.4 \%$ of non-survivors showing features of disseminated intravascular coagulation (DIC) during their hospital stay. ${ }^{9}$ This is mainly characterised by a prothrombotic diathesis with high VTE rate, $\mathrm{D}$-dimer, and fibrinogen levels..$^{10}$ There are several studies investigating D-dimers in COVID-19. Patients with severe COVID-19 had high D-dimer levels while those requiring ICU admission were shown to have even higher D-dimer levels, ${ }^{11}$ which was associated with development of acute respiratory distress syndrome (ARDS) and progression to death. ${ }^{12}$ A case-control study investigating VTE in COVID-19 patients showed that D-dimer was elevated in confirmed VTE cases and controls but higher levels were seen in confirmed VTE cases..$^{13} \mathrm{~A}$ pooled analysis by Lippi et al confirmed that D-dimer levels are higher in patients with severe COVID-19. However, it also highlighted heterogeneity across studies. ${ }^{14}$

Clinical observations from our hospital showed that a few critically unwell COVID-19 patients who developed VTE also had highly elevated levels of von Willebrand factor (vWF) and factor VIIIc. The aim of this study was to further explore markers of coagulopathy in patients with COVID-19 which could indicate or explain a tendency for VTE.

\section{Methods}

\section{Patients}

This study was carried out at the Brighton and Sussex University Hospitals NHS Trust. We identified 24 patients with COVID-19 who had undergone investigation for coagulopathy as part of their routine inpatient care. Included were consecutive patients who were admitted for active management of COVID-19 onto ITU, HDU and COVID wards of the hospital. Patients were admitted to the intensive care unit (ICU) or the high acuity ward and received standard care, according to the hospital's COVID-19 guidelines. Data collected up to 1 May 2020 were analysed.

\section{Materials}

Deep-vein thrombosis was diagnosed with doppler ultrasound, pulmonary embolism was diagnosed with $\mathrm{CT}$ pulmonary angiogram and hepatic veno-occlusive disease was diagnosed with contrast enhanced CT abdomen. Scans were performed only if there was a clinical suspicion of thrombosis and were reported by consultant radiologists. Blood samples were collected in $0.10^{9} \mathrm{M}$ sodium citrate tubes. All haemostasis assays were performed using a STA-R ${ }^{\circledR}$ MAX 2 coagulation analyser (Diagnostica Stago ${ }^{\circledR}$, Asnières-sur-Seine, France). The PT, APTT, fibrinogen and D-dimer were determined in plasma immediately after a single centrifugation $(2000 \mathrm{~g}$ for 10 min). PT and APTT were measured using STA ${ }^{\circledR}$-Neoplastine R and STA ${ }^{\circledR}$-Cephascreen, respectively (Stago). Quantitative determination of fibrinogen level was determined via the Clauss method, using the STA ${ }^{\circledR}$-Liquid Fib reagent (Stago). D-dimer was quantified by immunoturbidimetry using Liatest ${ }^{\circledR}$ D-Di PLUS reagent (Stago). Platelet-poor plasma (PPP) was then obtained by double centrifugation ( $2 \times 2000 \mathrm{~g}$ for $10 \mathrm{~min})$. PPP was frozen at $-80^{\circ} \mathrm{C}$ until use and thawed for $4 \mathrm{~min}$ in a $37^{\circ} \mathrm{C}$ water bath before the assays. FVIII activity was measured with a one-stage clotting assay using STA ${ }^{\circledR}$-C.K. Prest and STA ${ }^{\circledR}$-Immunodef VIII reagents
(Stago), calibrated to normal human plasma. VWF:Ag was quantified by immunoturbidimetry using Liatest ${ }^{\circledR}$ VWF:Ag reagent (Stago). VWF:RCo activity was quantified with STA ${ }^{\circledR}$-VWF:RCo (Stago), by means of a turbidimetric method based on platelet aggregation in presence of ristocetin.

\section{Statistics}

Statistical analysis was performed using GraphPad software. Comparison between two non-normally distributed variables was performed by Mann-Whitney test. Logistic regression was used when assessing correlation between clinical parameters and dichotomous variables (eg death/alive, blood group O/non-O, presence of VTE/no VTE). P-value $<0.05$ was considered statistically significant.

\section{Ethics}

This work was carried out as a service evaluation. Data collection and analysis were anonymised and secure with password protection.

\section{Results and discussion}

The rate of VTE was 25\% (6/24): pulmonary embolism (four patients), upper limb deep-vein thrombosis (one patient), hepatic veno-occlusive disease (one patient). Mortality rate was $16.7 \%$ $(4 / 24)$ and $75 \%$ were admitted to ICU. Of those admitted to ICU, $39 \%(7 / 18)$ were intubated and ventilated, while $61 \%(11 / 18)$ received level $1 /$ level 2 care. The following results are expressed in median (IQR). The age of COVID-19 positive patients was 65 (18) and they were predominately male (75\%). The INR and APTT ratio were both within the normal range, $1.1(0.1)$ and $1.3(0.3)$ respectively. Platelets were also within the normal range: 284 (251) $10^{9} / \mathrm{L}$. Fibrinogen was elevated at 7.9 (1.6) $\mathrm{g} / \mathrm{L}$ with an upper normal limit of $4.5 \mathrm{~g} / \mathrm{L}$. D-dimer were also increased 2.4 (2.02) ug/ $\mathrm{ml}$ with an upper normal limit of $0.5 \mathrm{ug} / \mathrm{ml}$. Factor VIII and von Willebrand factor antigen were considerably high, 279 (148) u/dL and 350 (131)\% respectively. 15 patients were also tested for antiphospholipid antibodies. Only two were positive for anti-cardiolipin IgM, while none were positive for anti-cardiolipin IgG or anti-B2 glycoprotein IgG.

Overall the results support the case that COVID-19 causes significant coagulopathy, with greatly increased vWF and factor VIIIc levels. This may be a result of endothelial damage. An explanation for the markedly increased vWF levels in COVID-19 patients could be that SARS-CoV-2 enters cells via the transmembrane protein ACE2. ACE2 is expressed on the surface of alveolar epithelial cells, and arterial and venous endothelial cells. ${ }^{15}$ The entry of the virus could contribute to inflammation and damage of endothelial cells causing release of prothrombotic mediators, primarily vWF from the Weibel-Palade storage bodies, and exposing underlying collagen to which VWF binds (Fig 1).

One patient in this series, with massive DVT and PE, and who exhibited extremely high vWF level $762 \mathrm{u} / \mathrm{dL}$, factor VIII $506 \%$ and RiCoF level $>200$, underwent more extensive analysis of coagulation parameters. This revealed raised factor IX 177\%, protein C 133\%, protein S $112 \%$ and reduced anti-thrombin level $74 \%$ (reference range $84-19 \%$ ). In addition, lupus anticoagulant testing was positive by DRVVT (correction 16.6\%) and dilute APTT (correction $37.7 \%$ ). Finally, and of particular interest, a reduced ADAMTS13 level of $49.7 \%$ (reference range $66-108$ ) was seen, suggesting loss of the 


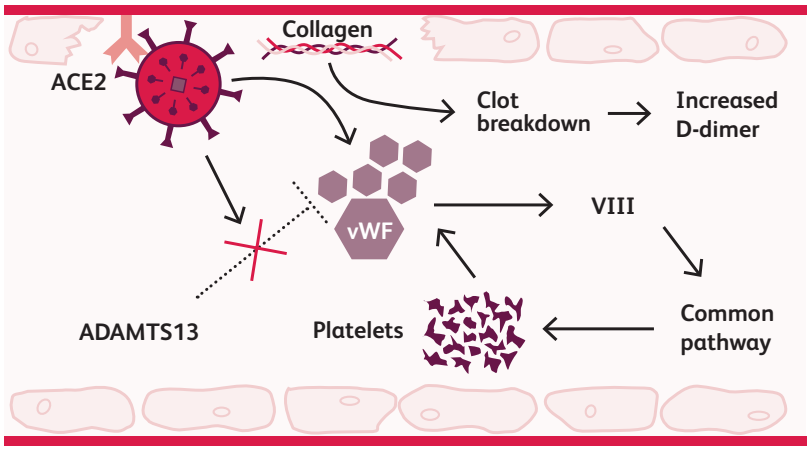

Fig 1. Schematic representation of how SARS-CoV-2 entry damages the endothelium, which subsequently results in vWF release mediating platelet aggregation and adhesion.

vWF cleaving protease that ordinarily degrades large vWF multimers and reduces its activity. We speculate that excess release of vWF seen in COVID-19 patients leads to depletion of ADAMTS13 and contributes to the prothrombotic state (Fig 1). Further studies are needed to measure ADAMT13 levels in a larger cohort of COVID-19 patients along with vWF multimer analysis to establish whether ultralarge multimers contribute to COVID-19 disease pathogenesis.

Helms et al recently published a multicenter prospective cohort study in France, assessing thrombotic risk in COVID-19 patients, which showed that vWF and VIIIc were considerably increased in COVID-19 patients. ${ }^{16}$ Our study, although smaller, has patients with similar demographic characteristics and confirms their findings in a British hospital setting. Detailed comparison of the two studies is described below (Table 1). Interestingly, while Helms et al examined those admitted to ICU and ventilated, our results extend these high wWF levels to those who are receiving level 1 (general ward-based)

Table 1. Clinical characteristics and laboratory parameters of patients with COVID-19 in our study and in the study by Helms et a ${ }^{16}$

$\begin{array}{lll} & \text { Helms et al } & \text { Our data } \\ \text { Number of patients } & 150 & 24 \\ \text { Age [median (IQR)] } & 63(53-71) & 65(55-72) \\ \text { Male [N (\%)] } & 122(81.3) & 18(75) \\ \text { Platelet [median (IQR)] } & 200(152-267) & 284(188-439) \\ \text { APTT ratio [median (IQR)] } & 1.2(1.1-1.3) & 1.3(1.1-1.4) \\ \text { INR [median (IQR)] } & 1.12(1.05-1.25) & 1.1(1.1-1.2) \\ \begin{array}{l}\text { D-dimer ug/ml } \\ \text { [median (IQR)] }\end{array} & 2.27(1.16-20) & 2.4(1.5-3.5) \\ \begin{array}{l}\text { Fibrinogen g/L } \\ \text { [median (IQR)] }\end{array} & 6.99(6.08-7.73) & 7.9(7.3-8.9) \\ \begin{array}{l}\text { Factor VIIIc u/dL (60-150) } \\ \text { [median (IQR)] }\end{array} & 341(258-416) & 279(251-363) \\ \begin{array}{l}\text { Von Willebrand factor } \\ \text { antigen \% (50-140) } \\ \text { [median (IQR)] }\end{array} & 455(350-521) & 350(302-433) \\ \end{array}$

and level 2 (high dependency) care. There was no statistically significant difference in VWF and factor VIIIc levels between ventilated versus non-ventilated patients. Panigada et al also reported that COVID-19 is characterised by hypercoagulability with a severe inflammatory state. Their results confirmed higher mean levels of vWf and factor VIIIc in their cohort, $529 \mathrm{U} / \mathrm{dl}$ and $297 \mathrm{U} / \mathrm{dl}$ respectively. ${ }^{17}$

It remains unclear whether this observed coagulopathy is the case for other infections, such as sepsis or viral pneumonias, as well. High levels of D-Dimers have been previously associated with 28-day mortality in patients presenting to the emergency department with sepsis. ${ }^{18}$ However, its prognostic value in sepsis has been questioned. ${ }^{19}$ Chen et al showed that patients with pneumococcal pneumonia were at higher risk for DVT and PE, which was highest during first 4 weeks but remained slightly elevated from 13 weeks to 2 years after acute infection. ${ }^{20}$ We compared our results to Kremer et al, who investigated vWF and related parameters in severe sepsis and septic shock. Their results showed that the median VWF antigen (\%) was significantly higher in patients (373) compared to controls (89) $(\mathrm{P}<0.001)$; however, this did not correlate with disease severity, organ dysfunction or outcome. ${ }^{21}$ Our results showed that the median vWF antigen was 342, which is very close to the vWF antigen present in acutely unwell patients with severe sepsis and septic shock. It is significantly higher than 89, which was the median vWF antigen measured in matched healthy controls by Kremer et al (Table 2). There are a few case reports on influenza and coagulopathy, but data on thrombotic microangiopathy is limited. Avnon et al reported that $25 \%$ of critically ill patients with influenza A/H1N1 had thrombotic events. ${ }^{22}$ Empirical systemic heparin anticoagulation in critically ill patients with H1N1 ARDS significantly reduced VTE incidence. ${ }^{23}$ Tsujii et al reported the case of a boy with heterozygous protein $\mathrm{S}$-deficiency, who was diagnosed with $\mathrm{H} 1 \mathrm{~N} 1$ influenza, whose plasma VWF was significantly elevated ( $299 \%$ of normal). ${ }^{24}$ Another case report by Akiyama et al also described 18-year-old female with $\mathrm{H} 1 \mathrm{~N} 1$ influenza whose plasma levels of VWF were remarkably raised $\left(191 \%\right.$ of normal). ${ }^{25}$ Lastly, varicella zoster virus has been linked to transient protein $\mathrm{C}$ and $\mathrm{S}$ deficiency, which was associated with thrombotic complications in children. ${ }^{26}$

Further analysis of our data showed that median vWF levels (\%) were significantly higher in patients that died (477) compared to the ones that remained alive (335) $(p=0.015)$. However, this needs further evaluation as the patients were not randomly selected and most remained alive by the end of the study ( $=18)$ (Fig 2). However, we did observe a positive correlation between age and vWF level with older COVID-19 patients tending to have higher $v W F$ levels with an approximate linear regression formula of vWF $=(4 \times$ age $)+100$ (Fig 3). This may contribute to the observed increased COVID-19 mortality rate in older individuals. Evaluation is needed to establish whether ethnic variations in vWF levels contribute to the different susceptibility rates amongst specific ethnic groups that is emerging in the current pandemic, although our data did not allow us to address this question.

Lastly, we investigated $A B O$ blood group given the known tendency for individuals with non-O blood to have higher vWF levels than individuals with group $\mathrm{O}$ blood. Five patients were excluded as the blood group was unknown. $68 \%$ of patients had non-O blood group (11/19 group $A$ and $2 / 19$ group $B$ ) compared 
Table 2. von Willebrand factor levels in patients with and without COVID-19 infection. Patients with COVID19, who have both critical and non-critical illness, have comparable vWF levels to patients with severe sepsis and septic shock in the absence of COVID-19

$\begin{array}{lllll}\text { Clinical diagnosis } & \begin{array}{l}\text { COVID-19 } \\ \text { (our data) }\end{array} & \text { Non-COVID-19 (data from Kramer et } \text { a }^{21} \text { ) } & \text { Septic shock } & \text { Controls } \\ \text { Number of patients } & 24 & 32 & 8 & 40 \\ \text { Male [N (\%)] } & 18(75) & 25(78) & 8(100) & \text { N/A } \\ \text { Age [median] } & 65 & 64 \text { (Males) } & 51.9 & \text { N/A } \\ \text { vWF Antigen \% [median] } & 350 & 70 \text { (Females) } & 373 & 89 \\ \text { Blood group O \% } & 32 & \text { N/A } & \text { N/A } & \text { N/A } \\ \text { Blood group A \% } & 58 & \text { N/A } & \text { N/A }\end{array}$

with $32 \%$ who had O blood group (6/19 group). Using NHS Blood Transfusion data for the expected frequency of non-O blood group (52\%) and O blood group ( $48 \%$ ) for the UK population, this difference is statistically significant ( $p=0.02$, Chi-square test). We also observed that $80 \%(4 / 5)$ of the patients who developed VTE were group A, while $20 \%(1 / 5)$ were group O $(p=0.22)$. Published literature suggests that patients with blood group A have higher rates of severe COVID-19 and mortality. ${ }^{27}$

\section{Conclusion}

This study provides a valuable insight into the coagulopathy that characterises COVID-19. D-dimers, fibrinogen, vWF factor and factor VIIIc are generally very high in COVID-19 patients and comparable to levels seen in severely unwell septic patients on ICU. This could be contributing to the hypercoagulable state with increased VTE rate, although we recognise that the correlation with elevated vWF levls does not necessarily imply mechanistic causation. We also demonstrate a tendency for more severe COVID-19 disease and VTE in patients with non-O blood group but recognise again

Von Willebrand factor levels in patients with COVID-19 pneumonia

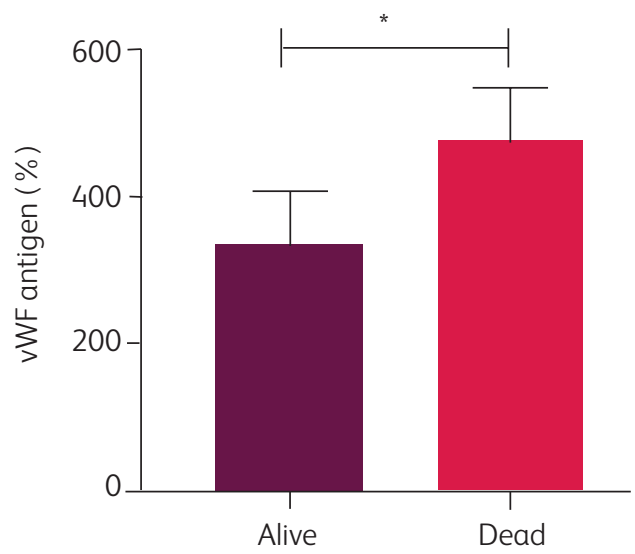

Fig 2. Percentage (\%) von Willebrand antigen in patients that have passed away or remained alive at the end of the study.
vWF Ag level vs age in COVID-19 patients

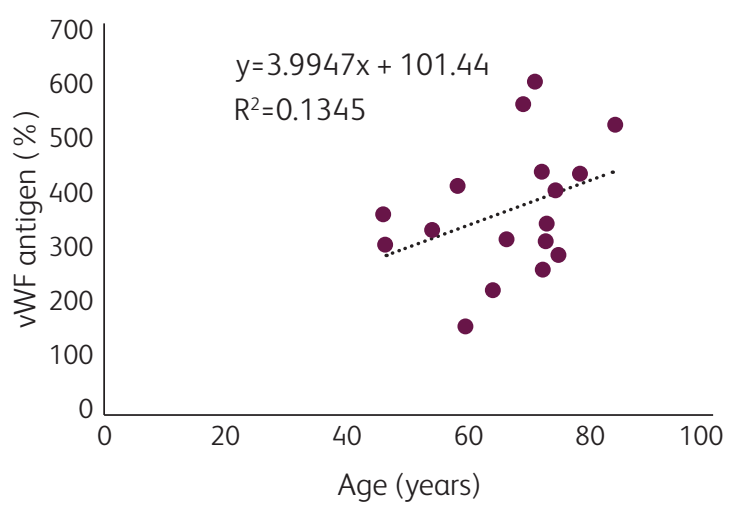

Fig 3. Scatter plot of vWF antigen (\%) in relation to age in COVID-19 patients.

that our data contains limited number of patients and therefore await other studies to confirm this result. Finally, we propose that vWF might serve as a simple prognostic measure of thrombotic risk in COVID-19 and potentially a significant contributor to disease pathogenesis.

\section{Acknowledgements}

The authors would like to acknowledge Lenka Mercer, Shawn Cotton and Dr Michael Tarzi, for their assistance in laboratory analysis.

\section{Funding}

Dr Eleni Ladikou and Dr Simon Stoneham are academic clinical fellows, funded by the National Institute for Health Research (NIHR). This publication presents independent research funded by the National Institute for Health Research (NIHR). The views expressed are those of the authors and not necessarily those of the NHS, the NIHR or the Department of Health and Social Care. 


\section{References}

1 Worldometer. COVID-19 coronavirus pandemic. www.worldometers. info/coronavirus/.

2 Yao XH, Li TY, He ZC et al. [A pathological report of three COVID-19 cases by minimally invasive autopsies]. Zhonghua Bing Li Xue Za Zhi 2020;49:E009.

3 Zhang T, Sun LX, Feng RE. [Comparison of clinical and pathological features between severe acute respiratory syndrome and coronavirus disease 2019]. Zhonghua Jie He He Hu Xi Za Zhi 2020;43:E040.

4 Fox SE, Akmatbekov A, Harbert JL et al. Pulmonary and cardiac pathology in Covid-19: the first autopsy series from New Orleans. MedRxiv 2020;2020.04.06.20050575.

5 Cade JF. High risk of the critically ill for venous thromboembolism. Crit Care Med 1982;10:448-50.

6 Songping C, Shuo C, Xiunan L, Shi L, Feng W. Prevalence of venous thromboembolism in patients with severe novel coronavirus pneumonia. J Thromb Haemost 2020, in press (doi: 10.1111/ jth.14830).

7 Danzi GB, Loffi M, Galeazzi G, Gherbesi E. Acute pulmonary embolism and COVID-19 pneumonia: a random association? Eur Heart J 2020;41:1858.

8 Tang N, Bai H, Chen X et al. Anticoagulant treatment is associated with decreased mortality in severe coronavirus disease 2019 patients with coagulopathy. J Thromb Haemost 2020;18:1094-9.

9 Tang N, Li D, Wang X, Sun Z. Abnormal coagulation parameters are associated with poor prognosis in patients with novel coronavirus pneumonia. J Thromb Haemost 2020;18:844-7.

10 Wang J, Hajizadeh N, Moore EE et al. Tissue plasminogen activator (tPA) treatment for COVID-19 associated acute respiratory distress syndrome (ARDS): a case series. J Thromb Haemost 2020;8:1752-5.

11 Huang C, Wang Y, Li X et al. Clinical features of patients infected with 2019 novel coronavirus in Wuhan, China. Lancet 2020;395:497-506.

12 Wu C, Chen X, Cai Y et al. Risk factors associated with acute respiratory distress syndrome and death in patients with coronavirus disease 2019 pneumonia in Wuhan, China. JAMA Intern Med 2020;180:1-11.

13 Stoneham S, Milne KM, Nuttall E, Frew GH, Sturrock BR, Sivaloganathan $\mathrm{H}$, et al. Thrombotic risk in COVID-19: a case series and case-control study. Clin Med 2020; 20:e76.

14 Lippi G, Favaloro EJ. D-dimer is associated with severity of coronavirus disease 2019: a pooled analysis. Thromb Haemost 2020;120:876-8.

15 Hamming I, Timens W, Bulthuis ML et al. Tissue distribution of ACE2 protein, the functional receptor for SARS coronavirus. A first step in understanding SARS pathogenesis. J Pathol 2004;203:631-7.

16 Helms ], Tacquard C, Severac F et al. High risk of thrombosis in patients in severe SARS-CoV-2 infection: a multicenter prospective cohort study. Intensive Care Med 2020, in press (doi: 10.1007/ s00134-020-06062-x).

17 Panigada M, Bottino N, Tagliabue P et al. Hypercoagulability of COVID-19 patients in intensive care unit. a report of thromboelastography findings and other parameters of hemostasis. J Thromb Haemost 2020;18:1738-42.

18 Rodelo JR, De la Rosa G, Valencia ML et al. D-dimer is a significant prognostic factor in patients with suspected infection and sepsis. Am J Emerg Med 2012;30:1991-9.

19 Semeraro F, Ammollo CT, Caironi P et al. D-dimer corrected for thrombin and plasmin generation is a strong predictor of mortality in patients with sepsis. Blood Transfus 2019, in press (doi: 10.2450/ 2019.0175-19).

20 Chen YG, Lin TY, Huang WY et al. Association between pneumococcal pneumonia and venous thromboembolism in hospitalized patients: A nationwide population-based study. Respirology 2015;20:799-804.

21 Kremer Hovinga JA, Zeerleder S, Kessler P et al. ADAMTS-13, von Willebrand factor and related parameters in severe sepsis and septic shock. J Thromb Haemost 2007;5:2284-90.

22 Avnon LS, Munteanu D, Smoliakov A, Jotkowitz A, Barski L. Thromboembolic events in patients with severe pandemic influenza A/H1N1. Eur J Intern Med 2015;26:596-8.

23 Obi AT, Tignanelli C], Jacobs BN et al. Empirical systemic anticoagulation is associated with decreased venous thromboembolism in critically ill influenza A H1N1 acute respiratory distress syndrome patients. J Vasc Surg Venous Lymphat Disord 2019;7:317-24.

24 Tsujii N, Nogami K, Yoshizawa H et al. Influenza-associated thrombotic microangiopathy with unbalanced von Willebrand factor and a disintegrin and metalloproteinase with a thrombospondin type 1 motif, member 13 levels in a heterozygous protein S-deficient boy. Pediatr Int 2016;58:926-9.

25 Akiyama R, Komori I, Hiramoto R et al. H1N1 influenza (swine flu)-associated thrombotic microangiopathy with a markedly high plasma ratio of von Willebrand factor to ADAMTS13. Intern Med 2011;50:643-7.

26 Nguyen P, Reynaud J, Pouzol P et al. Varicella and thrombotic complications associated with transient protein $\mathrm{C}$ and protein $\mathrm{S}$ deficiencies in children. Eur J Pediatr 1994;153:646-9.

27 Dai X. ABO blood group predisposes to COVID-19 severity and cardiovascular diseases. Eur ] Prev Cardiol 2020, in press (doi: 10.1177/2047487320922370).

Address for correspondence: Dr Timothy Chevassut, Brighton and Sussex Medical School, Medical Research Building, University of Sussex, Brighton, Surrey BN1 9PX, UK. Email: t.chevassut@bsms.ac.uk 